\title{
A SQUARE SHAPE OF THE GRAPH OF ITERATES OF MULTIFUNCTIONS: A COMPLETE CONTROLLABILITY RESULT
}

\author{
ARIE LEIZAROWITZ
}

(Communicated by William J. Davis)

\begin{abstract}
We consider a set valued function $T: K \rightarrow 2^{K}$ from a domain $K$ into itself. We look for conditions under which the graph of $T^{(n)}$ (the $n$th iterate of $T$ ) will be equal to $K \times K$ for some integer $n$. When the graph of $T$ is convex a sufficient (though not necessary) condition is that neither $T(x)$ nor $T^{-1}(x)$ are contained in the boundary of $K$ whenever $x$ is there. We show that a necessary and sufficient condition is that there are neither forward nor backward trajectories which remain in the boundary for all times. In the introduction, we remark on the significance of this problem for the study of infinite-horizon control systems.
\end{abstract}

\section{INTRODUCTION}

We consider a convex and compact set $K \subset R^{m}$ which has a nonempty interior, and a set valued function $T: K \rightarrow 2^{K}$ defined on the domain $K$ with values in the class of subsets of $K$. The graph of $T$, namely the set

$$
D=\{(x, y) \in K \times K: y \in T(x)\},
$$

is assumed to be a convex and compact subset of $R^{m} \times R^{m}$. Moreover, we assume that the projection on the second coordinate

$$
D_{Y}=\{y: \exists x \in K, y \in T(x)\},
$$

which we call the range of $T$, is equal to $K$. In this sense, $T$ is a mapping from $K$ onto $K$.

We consider iterates $T^{(n)}, n \geq 0$, of $T$ which are defined as follows. For every set $A \subset K$ we define

$$
T(A)=\bigcup_{x \in A} T(x) .
$$

Received by the editors December 13, 1988 and, in revised form, November 14, 1989.

1980 Mathematics Subject Classification (1985 Revision). Primary 52A20.

This research was supported by the Technion Vice President fund for research, the Albert Einstein Research Fund. 
We then define

$$
\begin{cases}T^{(0)}(x)=\{x\} & \forall x \in K, \\ T^{(n+1)}(x)=T\left(T^{(n)}(x)\right) & \forall x \in K \text { and } \forall n \geq 0 .\end{cases}
$$

Let $D_{n}, n \geq 1$, be the graph of $T^{(n)}$. We then ask the following question: Is there an integer $n \geq 1$ such that the graph of $T^{(n)}$ has a square shape, that is, such that $D_{n}=K \times K$ ?

The above system may be interpreted in terms of discrete time control systems. Let $K$ be the state space of the control system and let $T(x)$ be the states in $K$ to which the system can be steered in a unit time interval if it is at the state $x$ initially. Then the graph $D_{n}$ is composed of all the pairs $(x, y)$ such that the system can be steered from $x$ to $y$ in $n$ units of time. The question raised above is whether there exists an integer $n$ such that every state $x$ can be steered to every state $y$ in $n$ units of time.

The property that some $D_{n}$ has a square shape is important for the study of optimal control on infinite time intervals. (For details see Leizarowitz [3].) Thus the results of this paper can be applied to the study of various control systems, such as those considered e.g. in Leizarowitz [4] (for deterministic control systems) or Borkar [2] (for stochastic control problems).

Let the set valued function $T^{-1}$ be defined by

$$
T^{-1}(x)=\{y \in K: x \in T(y)\} \text {. }
$$

We then define $T^{(-n)}, n \geq 1$, as the $n$th iterate of $T^{-1}$.

We will prove that there exists a square shaped graph $D_{n}$ for some $n \geq 1$ provided that it is not the case that some boundary point can be steered only to boundary points, by either $T$ or $T^{-1}$. This sufficient condition is not necessary, as will be shown by a counterexample. However, a necessary and sufficient condition is that there is no point $x \in \partial K$ such that either $T^{(n)}(x) \subset \partial K$ or $T^{(-n)}(x) \subset \partial K$ holds for all $n \geq 0$ (where $\partial K$ is the boundary of $K$ ). This is our main result and appears as Theorem 3.3. Clearly the aforementioned sufficient condition is a corollary of this result. The sufficient condition is proved in the next section and the necessary and sufficient one is established in $\S 3$.

\section{THE SUFFICIENT CONDITION}

For the proof of the main result we will need the following lemma.

Lemma 2.1. Assume that the following holds:

$$
T(x) \not \subset \partial K \text { and } T^{-1}(x) \not \subset \partial K \text { for every } x \in \partial K \text {. }
$$

Then $D$ has nonempty interior and there exists an $x_{0} \in K$ such that $x_{0} \in$ int $T\left(x_{0}\right)$ (where int $A$ denotes the set of interior points of a set $A \subset R^{m}$ ).

Proof. The set valued mapping $x \rightarrow T(x)$ is continuous, hence by the Kakutani's fixed point theorem (see Aubin and Cellina [1, p. 85]), there exists a point $y \in K$ such that $y \in T(y)$. We may assume, without loss of generality, that 
$(0,0) \in D$ (since we may translate the point $y$ to the origin in $R^{m}$ and consider the set $K-y$ instead of $K$ ). If $D$ has an empty interior, then there are points $p, q \in R^{m},|p|+|q|>0$, such that $p \cdot x-q \cdot y=0$ for every $(x, y) \in D$. Thus, if $y \in T(x)$, then $q \cdot y=p \cdot x$. Let $\alpha=\max \{p \cdot x: x \in K\}$, $\beta=\max \{q \cdot y: y \in K\}$, and let $\bar{x}$ and $\bar{y}$ be such that $\bar{x}, \bar{y} \in K$ and $\alpha=p \cdot \bar{x}$ and $\beta=q \cdot \bar{y}$. Then for $y \in T(\bar{x})$, we have $\alpha=p \cdot \bar{x}=q \cdot y \leq \beta$, and if $x$ is such that $\bar{y} \in T(x)$, then $\alpha \geq p \cdot x=q \cdot \bar{y}=\beta$. Hence $\alpha=\beta$. The maximal common value $\alpha$ can be attained by $x \rightarrow p \cdot x$ and $y \rightarrow q \cdot y$ at boundary points of $K$ only, since both $p$ and $q$ are nonzero. Therefore, $\bar{x} \in \partial K$ and $T(\bar{x}) \subset \partial K$, which contradicts $(2.1)$ and establishes the claim that $D$ has a nonempty interior.

Let $\Sigma$ be the following subspace of $R^{m} \times R^{m}$ :

$$
\Sigma=\left\{(x, x): x \in R^{m}\right\} .
$$

Then the set $F=D \cap \Sigma$ of fixed points of $T$ is convex, compact, and nonempty since $(y, y) \in F$ (where $y$ is the fixed point guaranteed in the beginning of the proof). The second assertion of the lemma will follow once we have found an $x_{0}$ such that $\left(x_{0}, x_{0}\right) \in$ int $D$. But if the set $F$ is contained in the boundary of the convex set $D$, then by a separation theorem (see Rockafellar [5, Theorem 11.2 , p. 96]), there exists a $2 m-1$ dimensional subspace $\tilde{\Sigma}$ which contains $\Sigma$, and such that $D$ is on one side of $\tilde{\Sigma}$ (namely $D$ is contained in one of the closed half spaces which are determined by $\tilde{\Sigma})$. There are points $\lambda, \eta \in R^{m}$ such that

$$
\tilde{\Sigma}=\left\{(x, y) \in R^{m} \times R^{m}: \lambda \cdot x+\eta \cdot y=0\right\},
$$

and since $D$ is on one side of $\tilde{\Sigma}$, we have

$$
(x, y) \in D \Rightarrow \lambda \cdot x+\eta \cdot y \geq 0 .
$$

Since $\Sigma$ is contained in $\tilde{\Sigma}$, it follows that

$$
\lambda \cdot x+\eta \cdot x=0 \text { for every } x \in R^{m}
$$

hence $\eta=-\lambda$, so that the subspace $\tilde{\Sigma}$ is given by

$$
\{(x, y): \lambda \cdot(x-y)=0\},
$$

(where $\lambda \neq 0$ ). Therefore $\lambda \cdot(x-y) \geq 0$ for every $x \in K$ and $y \in T(x)$.

We now choose $\underline{x} \in \partial K$ such that $\lambda \cdot \underline{x}=\min _{x \in K} \lambda \cdot x$ and denote $\lambda \cdot \underline{x}$ by $\gamma$. Then, we claim we must have $T(\underline{x}) \subset \partial K$, because if $y \in T(\underline{x})$, then $\lambda \cdot y \leq \lambda \cdot \underline{x}=\gamma$, while the minimal value of $\gamma$ can be achieved only at boundary points of $K$. But $T(\underline{x}) \subset \partial K$ contradicts (2.1), which concludes the proof of the lemma.

We consider a point $x_{0}$ such that $x_{0} \in \operatorname{int} T\left(x_{0}\right)$ and define a sequence $\left\{Q_{n}\right\}_{n=0}^{\infty}$ of convex and compact subsets of $K$ by

$$
\left\{\begin{array}{l}
Q_{0}=\left\{x_{0}\right\} \\
Q_{n+1}=T\left(Q_{n}\right) \text { for } n \geq 0 .
\end{array}\right.
$$


Proposition 2.2. Consider the set $Q=\bigcup_{n=0}^{\infty} Q_{n}$, and assume that (2.1) holds. Moreover, assume that $D_{Y}$, the range of $T$, is equal to $K$. Then

$$
Q=K \text {. }
$$

Proof. The sequence $\left\{Q_{n}\right\}_{n=0}^{\infty}$ is increasing; hence $Q$ has a nonempty interior and $x_{0} \in$ int $Q$. Furthermore, the set $Q$ is invariant under $T$, that is

$$
T(Q) \subset Q .
$$

Assume that (2.3) is false. Then there exists an $x \in \partial K$ with $x \notin Q$. (Clearly $\partial K \subset Q$ implies (2.3).) Since the range of $T$ is $K$, there is a $y \in K$ such that $x \in T(y)$. It follows from (2.1) that we can choose $y \in \operatorname{int} K$. Also, (2.4) implies that $y \notin Q$. Let $l_{x}$ and $l_{y}$ be the straight line segments which connect $x_{0}$ with $x$ and $y$ respectively, and let $x^{\prime}$ and $y^{\prime}$ be the points where these lines meet the boundary $\partial Q$ of $Q$. (Thus $x^{\prime}$ is between $x_{0}$ and $x$, and $y^{\prime}$ is between $x_{0}$ and $y$.) We then have $y^{\prime}=\alpha x_{0}+(1-\alpha) y$ for some $0 \leq \alpha \leq 1$ and

$$
T\left(y^{\prime}\right)=T\left(\alpha x_{0}+(1-\alpha) y\right) \supset \alpha T\left(x_{0}\right)+(1-\alpha) T(y),
$$

which implies that

$$
\alpha x_{0}+(1-\alpha) x \in T\left(y^{\prime}\right) .
$$

We can write $x^{\prime}=\beta x_{0}+(1-\beta) x$ for some $0 \leq \beta \leq 1$. If we have

$$
\alpha<\beta \text {, }
$$

then it follows from $(2.5)$ that $T\left(y^{\prime}\right)$ contains points outside $\bar{Q}$, which contradicts (2.4). (Here we have denoted by $\bar{Q}$ the closure of $Q$ in $K$.) Thus, we have to show that (2.6) indeed holds for a certain choice of $x \in \partial K \backslash Q$.

For every $z \in \partial K$, let $z^{\prime}$ be the unique point in $\partial Q$ that lies on the straight line segment connecting $x_{0}$ and $z$. We write $z^{\prime}=\beta x_{0}+(1-\beta) z$ and consider the function $z \rightarrow \beta(z)$. It is continuous on $\partial K$ and we claim that it is not identically zero. Indeed if $x \in \partial K \backslash Q$ then there is $y \in \operatorname{int} K$ such that $x \in$ $T(y)$, hence $y \notin Q$. Let $\tilde{y} \in \partial K$ be on the line segment which connects $x_{0}$ and $y$ and such that $y$ is between $x_{0}$ and $\tilde{y}$. Then $\beta(\tilde{y})>0$.

We now return to the construction at the beginning of the proof and choose a point $x \in \partial K$ which maximizes $\beta(\cdot)$ on $\partial K$ :

$$
\beta(z) \leq \beta(x) \text { for all } z \in \partial K .
$$

We have $y \in \operatorname{int} K$ and we choose $\tilde{y} \in \partial K$ as above (namely $x_{0}, y$, and $\tilde{y}$ are colinear and $y$ is between $x_{0}$ and $\tilde{y}$ ). We then have

$$
\beta \geq \beta(\tilde{y})>\alpha
$$

with the inequality $\beta(\tilde{y})>\alpha$ being strict since $y \in \operatorname{int} K$. Thus (2.6) holds and the proposition is proved. 
Proposition 2.3. Assume that (2.1) holds. Then $Q_{p}=K$ for some $p \geq 1$.

Proof. Let the sequence $\left\{Q_{n}\right\}_{n=1}^{\infty}$ be defined by (2.2). We know by Proposition 2.2 that $K=\bigcup_{n=1}^{\infty} Q_{n}$. We claim that

$$
\bigcup_{n=0}^{\infty} \text { int } Q_{n} \supset \text { int } K \text {. }
$$

To prove this, let $x \in$ int $K$. If $x=x_{0}$, then $x \in$ int $Q_{1}$ (by the choice of $x_{0}$ ). If $x \neq x_{0}$, then we consider $x_{\varepsilon}=x_{0}+(1+\varepsilon)\left(x-x_{0}\right)$ and, for small values of $\varepsilon>0$, we have $x_{\varepsilon} \in$ int $K$. But then, $x_{\varepsilon} \in Q_{n}$ for some $n \geq 1$; hence $x \in$ int $Q_{n}$ for this $n$, which proves (2.7).

Let $\varphi: \partial K \rightarrow R^{1}$ be defined by

$$
\varphi(x)=\max \left\{\operatorname{dist}(y, \partial K): y \in T^{-1}(x)\right\} .
$$

It follows from the continuity of $T^{-1}$ that $\varphi(\cdot)$ is continuous on $\partial K$. Moreover, it follows from $(2.1)$ that $\varphi(x)>0$ for every $x \in \partial K$. Let

$$
\alpha=\min \{\varphi(x): x \in \partial K\} .
$$

Then $\alpha>0$ and for every $x \in \partial K$ there is $y \in$ int $K$ such that $\operatorname{dist}(y, \partial K) \geq$ $\alpha$. We consider the set

$$
K_{0}=\{x \in K: \operatorname{dist}(x, \partial K) \geq \alpha\}
$$

which is compact and is contained in int $K$. By (2.7), the collection int $\left.Q_{n}\right\}_{n=1}^{\infty}$ is an open covering of $K_{0}$, therefore, there is an integer $l \geq 1$ such that $K_{0} \subset Q_{l}$. But then

$$
Q_{l+1}=T\left(Q_{l}\right) \supset T\left(K_{0}\right) \supset \partial K
$$

which implies that $Q_{l+1} \supset K$ by the convexity of $Q_{l+1}$. Thus the assertion of the proposition holds with $p=l+1$.

Notice that the condition $D_{Y}=K$ is necessary in order for $D_{n}=K \times K$ to hold for some $n \geq 1$.

Theorem 2.4. Assume that (2.1) holds and that $D_{Y}=K$. Then there is an integer $n$ such that

$$
D_{n}=K \times K \text {. }
$$

That is, for every $x, y \in K$, we have

$$
y \in T^{(n)}(x) .
$$

Proof. It follows from Proposition 2.3 that there exists an integer $p>0$ such that $T^{(p)}\left(x_{0}\right)=K$.

We consider the set valued mapping $T^{-1}$ and observe that it satisfies the conditions of Proposition 2.3. We thus conclude that there is an integer $q>0$ such that $T^{(-q)}\left(x_{0}\right)=K$; namely, for every $x \in K$ we have $x_{0} \in T^{(q)}(x)$. But then (2.8) holds for every $x, y \in K$ with $n=p+q$, which concludes the proof of the theorem. 


\section{A NECESSARY AND SUFFICIENT CONDITION}

The sufficient condition of the previous section is not necessary as can be seen from the following example.

Example 3.1. Consider the set valued function $T$ which is defined on $[0,1]$ by

$$
T(x)=\left[\frac{1}{2}(1-x), \min \{1,2(1-x)\}\right] .
$$

Condition (2.1) is violated since $T(1)=\{0\} \subset \partial[0,1]$. However, the condition holds for the mapping $T^{(2)}$, hence there is an integer $n$ so that $D_{n}=[0,1] \times$ $[0,1]$.

The following result, which gives a necessary and sufficient condition, is an immediate consequence of Theorem 2.4 .

Proposition 3.2. Let $T$ be a set valued function from $K$ into itself whose graph $D$ is convex and compact. Then there exists a finite iterate $T^{(n)}, n \geq 1$ of $T$, whose graph $D_{n}$ satisfies

$$
D_{n}=K \times K
$$

if and only if the following two conditions hold:

(i) $\{y:(x, y) \in D$ for some $x \in K\}=K$, that is, the range of $T$ is $K$.

(ii) There exists an integer $p>0$ such that

$$
T^{(p)}(x) \not \subset \partial K \text { and } T^{(-p)}(x) \not \subset \partial K \quad \text { for every } x \in \partial K,
$$

that is, condition (2.1) holds for $T^{(p)}$.

Proof. The necessity of (i) is obvious. If $D_{n}=K \times K$ for some $n \geq 1$, then $T^{(n)}(x)=K$ and $T^{(-n)}(x)=K$ for every $x \in K$; in particular (ii) holds. The sufficiency part follows from Theorem 2.4 by considering the set valued mapping $T^{(p)}$.

The following is our main result, in which we strengthen the sufficiency part of Proposition 3.2 by replacing condition (ii) with

(iii) For every $x \in \partial K$, there exist integers $k, p>0$ such that $T^{(k)}(x) \not \subset$ $\partial K$, and $T^{(-p)}(x) \not \subset \partial K$.

Theorem 3.3. Let $T$ be a set valued function from $K$ into itself such that the graph $D$ of $T$ is convex and compact. Then there exists a finite iterate $T^{(n)}$, $n \geq 1$ of $T$, whose graph $D_{n}$ satisfies (3.1) if and only if $T$ is onto and no boundary point $x \in \partial K$ has the property that either its entire trajectory $\left\{T^{(n)}(x)\right\}_{n=0}^{\infty}$ or its entire backwards trajectory $\left\{T^{(-n)}(x)\right\}_{n=0}^{\infty}$ lies in $\partial K$.

Proof. The necessity part is clear. To prove the sufficiency part, we first show that for some integer, $p_{0}, T^{\left(p_{0}\right)}(x) \not \subset \partial K$ whenever $x \in \partial K$. If this claim were false, then there would be an $x_{p} \in \partial K$ such that $T^{(p)}\left(x_{p}\right) \subset \partial K$ for every $p \geq 1$. Hence we claim that

$$
T^{(i)}\left(x_{p}\right) \subset \partial K \quad \text { for all } 1 \leq i \leq p .
$$


Indeed, if (3.3) were false, then for some $1 \leq i<p$ we would have $y \in$ $T^{(i)}\left(x_{p}\right)$ and $y \in$ int $K$. But then $T(y) \not \subset \partial K$, since $T(y) \subset \partial K$ would imply, by the convexity of $D$, that $T(x) \subset \partial K$ for every $x \in \partial K$, which yields a contradiction to (iii). Therefore, $T^{(i+1)}\left(x_{p}\right) \not \subset \partial K$. We can now repeat the argument to conclude that $T^{(p)}\left(x_{p}\right) \not \subset \partial K$ that contradicts the choice of $x_{p}$.

The sequence $\left\{x_{p}\right\}_{p=1}^{\infty}$ in $\partial K$ contains a convergent subsequence $\left\{x_{p_{j}}\right\}_{j=1}^{\infty}$ such that

$$
x_{p_{j}} \rightarrow y \text { as } j \rightarrow \infty
$$

for some $y \in \partial K$. It follows from (3.3) that for every fixed $i \geq 1$ we have

$$
T^{(i)}\left(x_{p_{j}}\right) \subset \partial K
$$

for all large enough $j$. Hence, by letting $j \rightarrow \infty$ in (3.4), we get

$$
T^{(i)}(y) \subset \partial K, \quad \text { for every } i \geq 1 \text {. }
$$

This, however, contradicts (iii) and concludes the proof of the assertion that for some $p_{0} \geq 1$ we have $T^{\left(p_{0}\right)}(x) \not \subset \partial K$ for all $x \in \partial K$. It follows from the argument above that

$$
T^{(p)}(x) \not \subset \partial K \quad \text { for every } p \geq p_{0} \text { and all } x \in \partial K .
$$

Since the set valued function $T^{-1}$ also satisfies the conditions of the theorem, it follows that, analogous to (3.5), there exists an integer $p_{1}$ such that

$$
T^{(-p)}(x) \not \subset \partial K \quad \text { for every } p \geq p_{1} \text { and all } x \in \partial K \text {. }
$$

For $p=\max \left\{p_{0}, p_{1}\right\}$ the conditions of Proposition 3.2 are satisfied and the conclusion (3.1) follows. Thus the proof of the theorem is complete.

\section{ACKNOWLEDGMENT}

I would like to thank Professors Mete Soner and Ross Pinsky for several fruitful discussions concerning this work.

\section{REFERENCES}

1. J. P. Aubin and A. Cellina, Differential inclusions, Springer-Verlag, New York, 1984.

2. V. S. Borkar, On minimum cost per unit time control of Markov chains, SIAM J. Control Optim. 22 (1984), 965-978.

3. A. Leizarowitz, Infinite horizon autonomous systems with unbounded cost, Appl. Math. Optim. 13 (1985), 19-43.

4. __ Optimal control of infinite horizon deterministic control problems, Appl. Math. Optim. 17 (1988), 61-79.

5. R. T. Rockafellar, Convex analysis, Princeton University Press, Princeton, NJ, 1970.

Department of Mathematics, Technion, Haifa 32000, IsRael 